\title{
Mass Equivalent Pantographs for Synthesis of Balanced Focal Mechanisms
}

\author{
Volkert van der Wijk
}

\begin{abstract}
Force balance is an important property in the design of high-speed high precision machinery to reduce base vibrations and also for the design of inherently safe large movable structures. This paper presents the synthesis of inherently balanced overconstrained focal mechanisms with mass equivalent pantographs. It is shown how pantograph linkages can be combined into an overconstrained but movable linkage by connecting them in their similarity points. With mass equivalent modeling the force balance conditions are derived for which the common center of mass is in the focal point for any motion. As examples Burmester's focal mechanism is investigated for balance and a new balanced focal mechanism of three mass equivalent pantographs is presented.
\end{abstract}

Key words: focal mechanism, pantograph, Burmester, force balance, mass equivalence

\section{Introduction}

In robotics, dynamic (shaking) force balance is an important property for high-speed motion with minimal base vibrations [4]. Since force balanced mechanisms are statically balanced too, it is also an useful property for large moving structures for save motion with minimal effort.

A problem of common approaches to balance pre-existing mechanisms is that generally a multitude of counter-masses is required [1,9], leading to unpractical designs with a significant increase of mass and inertia [6]. Instead, a reversed approach was presented where balanced mechanisms are synthesized from inherently balanced linkage architectures [4]. These linkages consist solely of the essential

V. van der Wijk

Centre for Robotics Research, Dep. of Informatics, Fac. of Natural and Mathematical Sciences, King's College London, Strand, London (UK); e-mail: Volkert.vanderWijk@kcl.ac.uk 
kinematic and mass properties for balance. With this method a variety of new advantageous inherently balanced mechanism solutions were found among which the first high-speed dynamically balanced parallel manipulator that was successfully built and tested [8].

With inherent balancing it is also possible to synthesize balanced mechanism solutions from overconstrained inherently balanced linkage architectures [7]. These architectures have more links than kinematically needed. This gives the designer the freedom to select links to keep or eliminate to obtain a normally constrained balanced mechanism solution. Also more solutions can potentially be found.

The goal of this paper is to investigate focal mechanisms, which are overconstrained and movable, for inherent balance. The focal mechanism of Burmester [2] - the cognate of Kempe's focal mechanism - can be regarded a combination of two pantographs [3]. It is shown how these two pantograph linkages can be combined by connecting them in their similarity points. For force balance the two pantographs need to be mass equivalent with a model of which the common center of mass $(\mathrm{CoM})$ is in the focal point. The conditions for this are derived. In addition also a new inherently balanced focal mechanism of three combined pantographs is presented at the end.

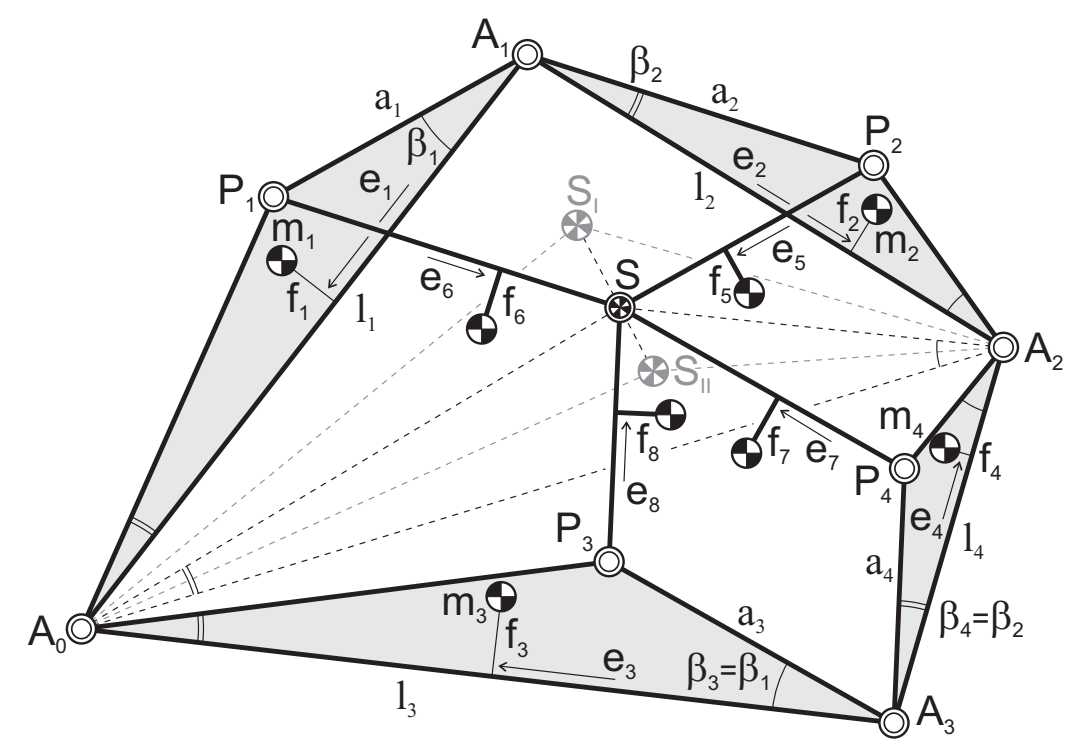

Fig. 1 Burmester's focal mechanism of two pantograph linkages connected in their similarity points $A_{0}, A_{2}$, and $S . S$ is the focal point and is the common CoM of all elements for force balance. 


\section{$2 \mathrm{CoM}$ in focal point of Burmester's focal mechanism}

Figure 1 shows Burmester's focal mechanism which consists of the two pantograph linkages $P_{1} A_{1} P_{2} S$ - with link lengths $l_{1}, l_{2}, a_{1}$, and $a_{2}$ - and $P_{3} A_{3} P_{4} S$ - with link lengths $l_{3}, l_{4}, a_{3}$, and $a_{4}$ - that are connected with revolute pairs in the similarity points $A_{0}, A_{2}$, and $S$. This linkage is two times overconstrained yet movable since both pantographs are similar, i.e. elements $A_{0} A_{1} P_{1} \sim A_{1} A_{2} P_{2} \sim A_{0} A_{3} P_{3} \sim A_{3} A_{2} P_{4}$ with angles $\beta_{1}$ and $\beta_{2}$. These four triangular elements are also similar to triangle $A_{0} A_{2} S$ for any motion of the mechanism. Both pairs of opposite internal four-bars are reflected similar to one another, with one pair being parallelograms.

When, for example, for the upper pantograph $a_{1}, a_{2}, l_{1}$, and $\beta_{1}$ are given, $l_{2}$ and $\beta_{2}$ can be calculated as

$$
\begin{array}{r}
\lambda_{1}^{S}=1-\frac{a_{1}}{l_{1}} \cos \beta_{1}, \quad \lambda_{2}^{S}=\frac{a_{1}}{l_{1}} \sin \beta_{1} \\
\beta_{2}=\tan ^{-1} \frac{\lambda_{2}^{S}}{\lambda_{1}^{S}}, \quad l_{2}=\frac{a_{2}}{\lambda_{1}^{S}} \cos \beta_{2}=\frac{a_{2}}{\lambda_{2}^{S}} \sin \beta_{2}
\end{array}
$$

with $\lambda_{1}^{S}$ and $\lambda_{2}^{S}$ the constant similarity parameters of the four triangular elements and triangle $A_{0} A_{2} S$. When, subsequently, for the lower pantograph $l_{3}$ and $l_{4}$ are given, $a_{3}$ and $a_{4}$ can be calculated as

$$
a_{3}=\left(1-\lambda_{1}^{S}\right) \frac{l_{3}}{\cos \beta_{1}}=\lambda_{2}^{S} \frac{l_{3}}{\sin \beta_{1}}, \quad a_{4}=l_{4} \frac{\lambda_{1}^{S}}{\cos \beta_{2}}=l_{4} \frac{\lambda_{2}^{S}}{\sin \beta_{2}}
$$

These parameters can also be obtained from the similarity conditions of the four triangular elements which write

$$
\frac{a_{1}}{l_{1}}=\frac{a_{3}}{l_{3}}, \quad \frac{a_{2}}{l_{2}}=\frac{a_{4}}{l_{4}}
$$

In Fig. 1 each of the eight links $i$ has a mass $m_{i}$ of which the CoM is defined with parameters $e_{i}$ and $f_{i}$ as illustrated. The aim is to design the mechanism such that the common CoM of all elements is in focal point $S$ for any motion. Then the mechanism is inherently force balanced with respect to the focal point.

The force balance conditions describe how the CoMs of each element are related for balance. These conditions can be found by mass equivalent modeling with real and virtual equivalent masses [4,5]. With mass $m_{I}=m_{1}+m_{2}+m_{5}+m_{6}$ of upper pantograph $P_{1} A_{1} P_{2} S$ and mass $m_{I I}=m_{3}+m_{4}+m_{7}+m_{8}$ of lower pantograph $P_{3} A_{3} P_{4} S$ the total mass of the focal mechanism can be written as $m_{t o t}=m_{I}+m_{I I}$. The common CoM of the upper pantograph is denoted $S_{I}$ and the common CoM of the lower pantograph is denoted $S_{I I}$. With similarity points $A_{0}$ and $A_{2}$ these two points form two triangles as well which also have to remain similar for any motion. For force balance then each pantograph is mass equivalent to a 2-DoF mass equivalent model with the conditions [5] 


$$
\begin{array}{r}
m_{I}^{a}=m_{I}\left(1-\lambda_{1}^{I}\right), \quad m_{I}^{b}=m_{I} \lambda_{1}^{I}, \quad m_{I}^{c}=m_{I} \lambda_{2}^{I} \\
m_{I I}^{a}=m_{I I}\left(1-\lambda_{1}^{I I}\right), \quad m_{I I}^{b}=m_{I I} \lambda_{1}^{I I}, \quad m_{I I}^{c}=m_{I I} \lambda_{2}^{I I}
\end{array}
$$

with $\lambda_{1}^{I}$ and $\lambda_{2}^{I}$ the similarity parameters of triangle $A_{0} A_{2} S_{I}, \lambda_{1}^{I I}$ and $\lambda_{2}^{I I}$ the similarity parameters of triangle $A_{0} A_{2} S_{I I}$, and real equivalent masses $m_{j}^{a}$ and $m_{j}^{b}$ and virtual equivalent mass $m_{j}^{c}$ of each pantograph $j$. For the upper pantograph in Fig. 2a, Fig. 2b shows the 2-DoF mass equivalent model adapted from [5]. Essentially the virtual equivalent mass determines the link CoMs relative to the lines connecting the joints, i.e. the values of parameters $f_{i}$, whereas the real equivalent masses determine the link CoMs along the lines connecting the joints, i.e. the values of parameters $e_{i}$.

To have the common CoM in the focal point, the sum of the mass equivalent models of the two pantographs should equal the mass equivalent model of the complete mechanism. This can be written as $m_{I} \lambda_{1}^{I}+m_{I I} \lambda_{1}^{I I}=m_{\text {tot }} \lambda_{1}^{S}$ and $m_{I} \lambda_{2}^{I}+m_{I I} \lambda_{2}^{I I}=m_{t o t} \lambda_{2}^{S}$. The resulting model is similar to Fig. $2 \mathrm{~b}$ but with each equivalent mass replaced with the sum of the equivalent masses of the two pantograph models as $m^{a}=m_{I}^{a}+m_{I I}^{a}, m^{b}=m_{I}^{b}+m_{I I}^{b}$, and $m^{c}=m_{I}^{c}+m_{I I}^{c}$. The conditions for the mass equivalent model of the complete mechanism then are written as

$$
m^{a}=m_{t o t}\left(1-\lambda_{1}^{S}\right), \quad m^{b}=m_{t o t} \lambda_{1}^{S}, \quad m^{c}=m_{t o t} \lambda_{2}^{S}
$$

The force balance conditions for each pantograph can be derived from the linear momentum equations of each DoF individually where the linear momentum of the mass equivalent model must equal the linear momentum of the real pantograph, similar as for the dyads in [5]. Figure 3a shows the mass motions of DoF 1 of the upper pantograph where link $A_{1} A_{2}$ is fixed and link $A_{0} A_{1}$ rotates about $A_{1}$ with angle $\theta_{I 1}$. The mass motion of the pantograph for this DoF is shown on the right with a compact Equivalent Linear Momentum System (ELMS) where all masses

(a)

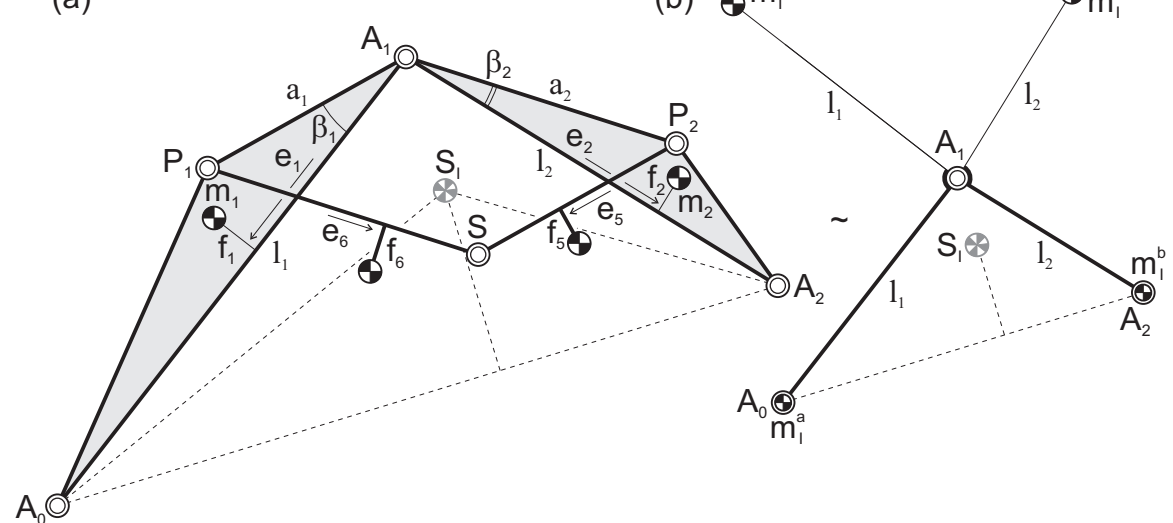

Fig. 2 For force balance (a) each pantograph must be mass equivalent to the (b) 2-DoF mass equivalent model, here shown for the upper pantograph with CoM in $S_{I}$. 
are projected on element $A_{0} A_{1}$. Figure $3 \mathrm{~b}$ shows the mass motions of DoF 2 where link $A_{1} A_{0}$ is fixed and link $A_{1} A_{2}$ rotates about $A_{1}$ with angle $\theta_{I 2}$. Also here the mass motion of the pantograph for this DoF is shown on the right with a compact ELMS where all masses are projected on element $A_{1} A_{2}$. The linear momentum $L_{1}$ and $L_{2}$ of these individual motions can be written with respect to their relative reference frames $x_{I 1} y_{I 1}$ and $x_{I 2} y_{I 2}$, which are aligned with lines $A_{0} A_{1}$ and $A_{2} A_{1}$, respectively, as

$$
\begin{aligned}
& \frac{\bar{L}_{1}}{\dot{\theta}_{I 1}}=\left[\begin{array}{c}
m_{I}^{a} l_{1} \\
-m_{I}^{c} l_{1}
\end{array}\right]=\left[\begin{array}{c}
m_{1} e_{1}+m_{5}\left(e_{5} \cos \beta_{1}+f_{5} \sin \beta_{1}\right)+m_{6} a_{1} \cos \beta_{1} \\
-m_{1} f_{1}-m_{5}\left(e_{5} \sin \beta_{1}-f_{5} \cos \beta_{1}\right)-m_{6} a_{1} \sin \beta_{1}
\end{array}\right] \\
& \frac{\bar{L}_{2}}{\dot{\theta}_{I 2}}=\left[\begin{array}{c}
m_{I}^{b} l_{2} \\
m_{I}^{c} l_{2}
\end{array}\right]=\left[\begin{array}{c}
m_{2} e_{2}+m_{5} a_{2} \cos \beta_{2}+m_{6}\left(e_{6} \cos \beta_{2}+f_{6} \sin \beta_{2}\right) \\
m_{2} f_{2}+m_{5} a_{2} \sin \beta_{2}+m_{6}\left(e_{6} \sin \beta_{2}-f_{6} \cos \beta_{2}\right)
\end{array}\right]
\end{aligned}
$$

These equations result in the four force balance conditions

$$
\begin{aligned}
m_{I}^{a} l_{1} & =m_{1} e_{1}+m_{5}\left(e_{5} \cos \beta_{1}+f_{5} \sin \beta_{1}\right)+m_{6} a_{1} \cos \beta_{1} \\
m_{I}^{c} l_{1} & =m_{1} f_{1}+m_{5}\left(e_{5} \sin \beta_{1}-f_{5} \cos \beta_{1}\right)+m_{6} a_{1} \sin \beta_{1} \\
m_{I}^{b} l_{2} & =m_{2} e_{2}+m_{5} a_{2} \cos \beta_{2}+m_{6}\left(e_{6} \cos \beta_{2}+f_{6} \sin \beta_{2}\right) \\
m_{I}^{c} l_{2} & =m_{2} f_{2}+m_{5} a_{2} \sin \beta_{2}+m_{6}\left(e_{6} \sin \beta_{2}-f_{6} \cos \beta_{2}\right)
\end{aligned}
$$

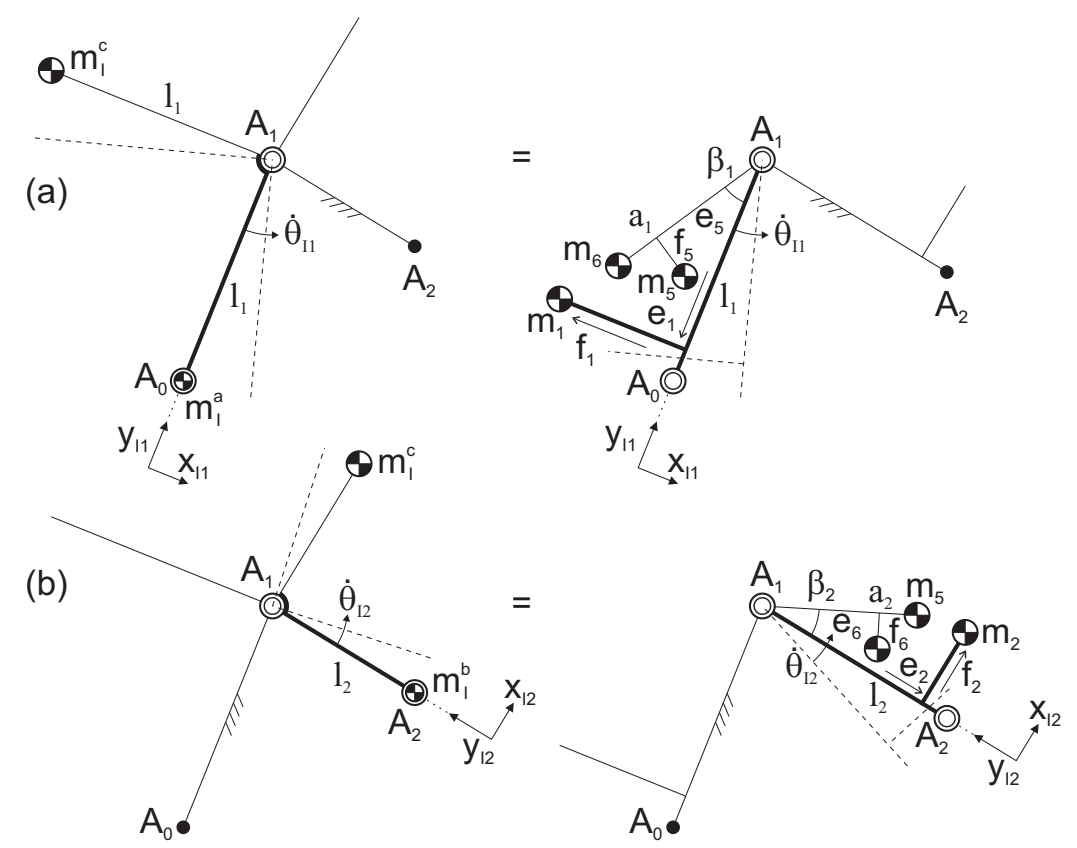

Fig. 3 The force balance conditions are derived from the linear momentum equations of each DoF individually which are equal for the mass equivalent model (left) and the real pantograph (right, here shown as compact Equivalent Linear Momentum Systems). 
For the other pantograph the force balance conditions can be derived similarly as

$$
\begin{aligned}
& m_{I I}^{a} l_{3}=m_{3} e_{3}+m_{7}\left(e_{7} \cos \beta_{1}+f_{7} \sin \beta_{1}\right)+m_{8} a_{3} \cos \beta_{1} \\
& m_{I I}^{c} l_{3}=m_{3} f_{3}+m_{7}\left(e_{7} \sin \beta_{1}-f_{7} \cos \beta_{1}\right)+m_{8} a_{3} \sin \beta_{1} \\
& m_{I I}^{b} l_{4}=m_{4} e_{4}+m_{7} a_{4} \cos \beta_{2}+m_{8}\left(e_{8} \cos \beta_{2}+f_{8} \sin \beta_{2}\right) \\
& m_{I I}^{c} l_{4}=m_{4} f_{4}+m_{7} a_{4} \sin \beta_{2}+m_{8}\left(e_{8} \sin \beta_{2}-f_{8} \cos \beta_{2}\right)
\end{aligned}
$$

These are the 8 general force balance conditions of the focal mechanism in Fig. 1 for which the common CoM is in the focal point $S$. For example, from the first four equations the equivalent masses $m_{I}^{a}, m_{I}^{b}$, and $m_{I}^{c}$ may be found to subsequently calculate with Eqs. (5) the equivalent masses $m_{I I}^{a} m_{I I}^{b}$, and $m_{I I}^{c}$ to be used in the latter four balance conditions. It is also possible to initially choose values for $m_{I}^{a} m_{I}^{b}$, and $m_{I}^{c}$. Then for instance from the first four equations $e_{5}, f_{5}, e_{6}$, and $f_{6}$ can be derived as

$$
\begin{aligned}
& e_{5}=\frac{\sin \beta_{1}\left(m_{I}^{c} l_{1}-m_{1} f_{1}-m_{6} a_{1} \sin \beta_{1}\right)+\cos \beta_{1}\left(m_{I}^{a} l_{1}-m_{1} e_{1}-m_{6} a_{1} \cos \beta_{1}\right)}{m_{5}} \\
& f_{5}=\frac{\sin \beta_{1}\left(m_{I}^{a} l_{1}-m_{1} e_{1}-m_{6} a_{1} \cos \beta_{1}\right)-\cos \beta_{1}\left(m_{I}^{c} l_{1}-m_{1} f_{1}-m_{6} a_{1} \sin \beta_{1}\right)}{m_{5}} \\
& e_{6}=\frac{\sin \beta_{2}\left(m_{I}^{c} l_{2}-m_{2} f_{2}-m_{5} a_{2} \sin \beta_{2}\right)+\cos \beta_{2}\left(m_{I}^{b} l_{2}-m_{2} e_{2}-m_{5} a_{2} \cos \beta_{2}\right)}{m_{6}} \\
& f_{6}=\frac{\sin \beta_{2}\left(m_{I}^{b} l_{2}-m_{2} e_{2}-m_{5} a_{2} \cos \beta_{2}\right)-\cos \beta_{2}\left(m_{I}^{c} l_{2}-m_{2} f_{2}-m_{5} a_{2} \sin \beta_{2}\right)}{m_{6}}
\end{aligned}
$$

\section{Focal mechanism of three pantographs}

In general it is possible to synthesize a variety of inherently force balanced focal linkages by combining multiple mass equivalent pantographs in the same way as in the previous section. Figure 4 shows a new focal mechanism that is composed of the three pantographs $P_{1} A_{1} P_{2} S, P_{3} A_{3} P_{4} S$, and $P_{5} A_{5} P_{6} S$ which are connected in similarity points $A_{0}, A_{2}, A_{4}$, and $S$ where $S$ is the focal point. The resulting linkage is four times overconstrained yet movable. Also here each pantograph has similar triangular elements and a similar triangle of the similarity points. However in this case the pantographs differ from one another, e.g. the triangular elements of pantograph $P_{1} A_{1} P_{2} S$ are not similar to the triangular elements of the other pantographs. In fact the focal mechanism is a combination of the three different triangles $A_{0} A_{2} S$, $A_{2} A_{4} S$, and $A_{0} A_{4} S$ that together form the triangle $A_{0} A_{2} A_{4}$. For each pantograph the dimensions of the elements can be calculated with Eqs. (1) with for each pantograph different $\lambda^{S}$ parameters. For two pantographs the $\lambda^{S}$ parameters can be chosen independently such that with the triangle $A_{0} A_{2} A_{4}$ the third is determined.

The approach to derive the force balance conditions for which the common CoM is in focal point $S$ is similar to Burmester's focal mechanism. Here the mechanism 
can be considered a combination of three mass equivalent models with each a mass $m_{I}, m_{I I}$, and $m_{I I I}$ with CoMs in $S_{I}, S_{I I}$, and $S_{I I I}$, respectively as illustrated in Fig. 5a. For each pantograph the force balance conditions can be found with Eqs. (6). The equivalent masses $m_{j}^{a}, m_{j}^{b}$, and $m_{j}^{c}$ of each mass equivalent model are defined according to Eqs. (4). The mass equivalent model of the complete focal mechanism has real equivalent masses $m_{I}^{a}+m_{I I I}^{b}$ in $A_{0}, m_{I}^{b}+m_{I I}^{a}$ in $A_{2}$, and $m_{I I}^{b}+m_{I I I}^{a}$ in $A_{4}$ and it has virtual equivalent masses $m_{I}^{c}$ about $S_{I}, m_{I I}^{c}$ about $S_{I I}$, and $m_{I I I}^{c}$ about $S_{I I I}$ as illustrated. Figure $5 \mathrm{~b}$ shows the unified mass equivalent model of the complete focal mechanism from which the conditions for which $S$ is the CoM of this model can be derived as

$$
\begin{aligned}
& \left(m_{I I}^{b}+m_{I I I}^{a}\right) d_{3}+\left(m_{I}^{b}+m_{I I}^{a}\right) d_{1} \cos \psi_{1}=m_{t o t} h_{1} \\
& \left(m_{I}^{b}+m_{I I}^{a}\right) d_{1} \sin \psi_{1}=m_{t o t} h_{2} \\
& m_{I I}^{c} d_{3}-m_{I I}^{c} d_{2} \cos \psi_{3}-m_{I}^{c} d_{1} \cos \psi_{1}=0 \\
& m_{I}^{c} d_{1} \sin \psi_{1}-m_{I I}^{c} d_{2} \sin \psi_{3}=0
\end{aligned}
$$

with total mass $m_{\text {tot }}=m_{I}+m_{I I}+m_{I I I}$ and with the CoM in $S$ defined with respect to $A_{0} A_{4}$ by $h_{1}$ and $h_{2}$.

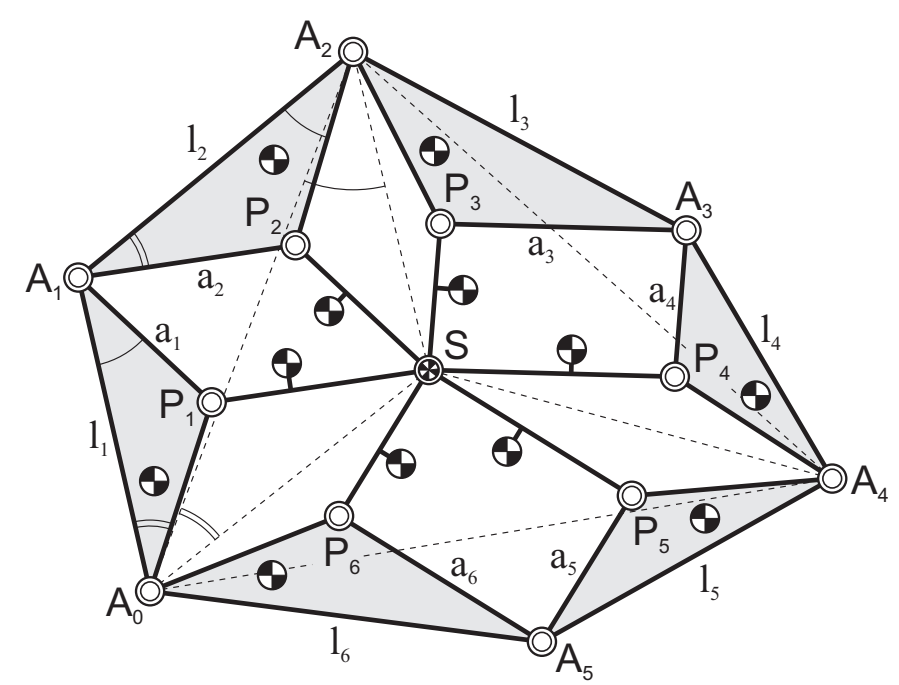

Fig. 4 Focal mechanism of three pantograph linkages connected in their similarity points $A_{0}, A_{2}$, $A_{4}$, and $S . S$ is the focal point and is the common CoM of all elements for force balance. 


\section{Discussion and conclusion}

The inherent force balance of Burmester's focal mechanism was investigated and the force balance conditions were derived. It was shown that for balancing the focal mechanism can be considered composed of two mass equivalent pantographs. Combination of the mass equivalent models of the pantographs then results in one mass equivalent model of which the center of mass is in the focal point.

It was also shown how with three mass equivalent pantographs a new focal mechanism could be designed. In general, by combining multiple mass equivalent pantographs a variety of inherently balanced focal mechanisms can be synthesized. Uni-

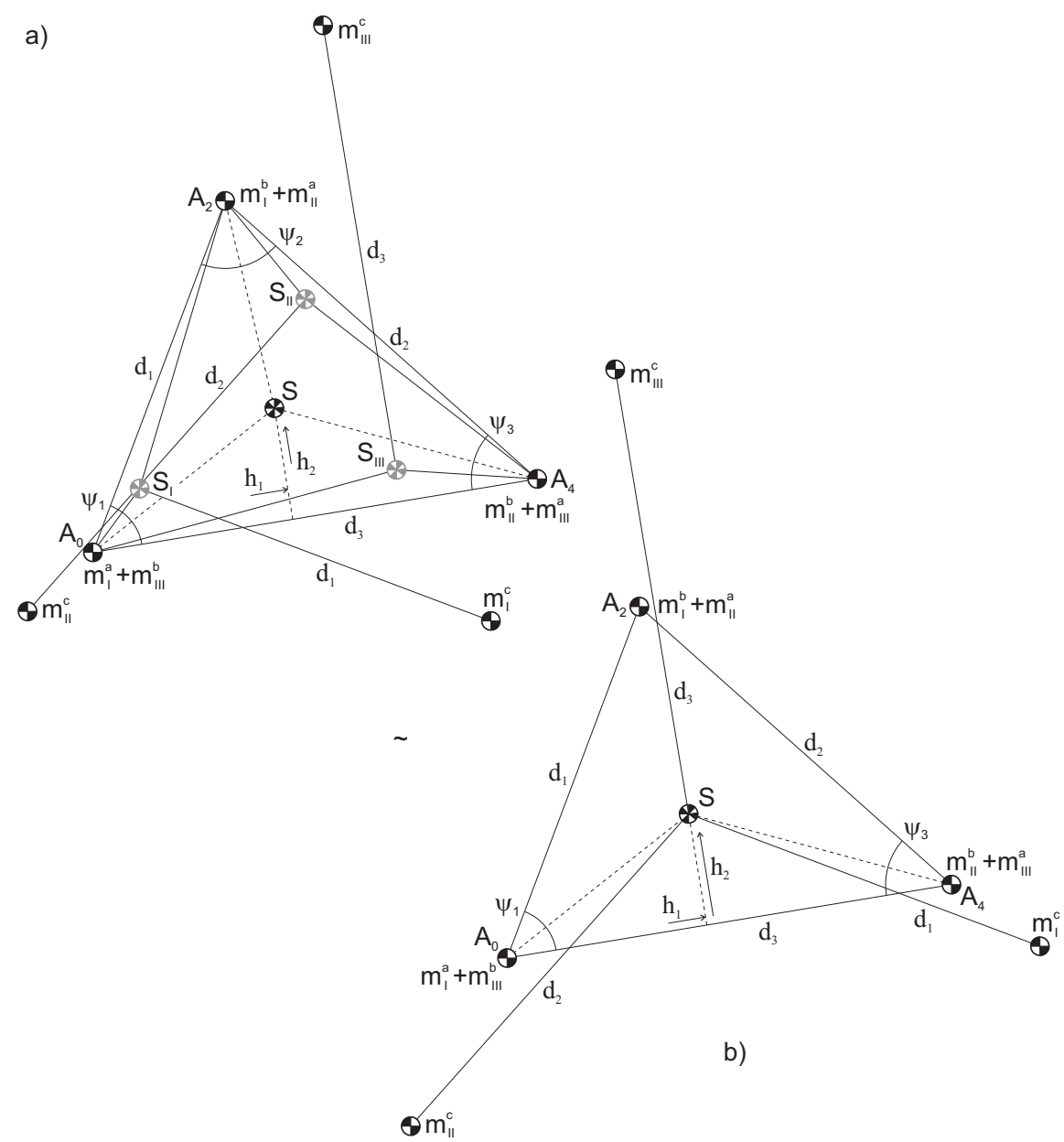

Fig. 5 a) Combination of the three mass equivalent models with their equivalent masses. The common CoM of the focal mechanism is the CoM of this combined mass equivalent model; b) The unified mass equivalent model of the focal mechanism of which $S$ is the CoM. 
fying the mass equivalent models of all pantographs then results in a single mass equivalent model of which the center of mass is in the focal point.

Parameters $a_{i}$ are the principal dimensions of the focal mechanism when its common center of mass is in the focal point. When the center of mass of an individual pantograph is in the focal point, then $a_{i}$ are also the principal dimensions of this individual pantograph.

Although in Burmester's focal mechanism the two pantographs are in opposite branch, this is not required from the force balance conditions. This means that for force balance one of the pantographs or both of them may also be in the other branch, which means that they could also appear as being on top of one another.

\section{Acknowledgement}

This publication was financially supported by the Niels Stensen Fellowship.

\section{References}

1. Briot, S., Bonev, I.A., Gosselin, C.M., Arakelian, V.: Complete shaking force and shaking moment balancing of planar parallel manipulators with prismatic pairs. Multi-body Dynamics 223(K), 43-52 (2009)

2. Burmester, L.: Die brennpunktmechanismen. Zeitschrift für Mathematik und Physik 38, 193 223 and 3 appendices (1893)

3. Dijksman, E.A.: Motion Geometry of Mechanisms. Cambridge University Press (1979)

4. Van der Wijk, V.: Methodology for analysis and synthesis of inherently force and momentbalanced mechanisms - theory and applications (dissertation). University of Twente (free download: http://dx.doi.org/10.3990/1.9789036536301) (2014)

5. Van der Wijk, V.: Mass equivalent dyads. In: S. Bai and M. Ceccarrelli (eds.), Recent Advances in Mechanism Design for Robotics MMS 33, 35-45 (2015). Springer.

6. Van der Wijk, V., Demeulenaere, B., Gosselin, C., Herder, J.L.: Comparative analysis for low-mass and low-inertia dynamic balancing of mechanisms. Mechanisms and Robotics 4(3, 031008) (2012)

7. Van der Wijk, V., Herder, J.L.: Inherently balanced $4 \mathrm{R}$ four-bar based linkages. In: Lenarčič, J. and Husty, M. (Eds.), Latest Advances in Robot Kinematics, Proc. of the IFToMM 13th Int. Symposium on Advances in Robot Kinematics pp. 309-316 (2012). Springer, ISBN 978-94007-4619-0

8. Van der Wijk, V., Krut, S., Pierrot, F., Herder, J.L.: Design and experimental evaluation of a dynamically balanced redundant planar 4-RRR parallel manipulator. I.J. of Robotics Research 32(6), 744-759 (2013)

9. Wu, Y., Gosselin, C.M.: Design of reactionless 3-DOF and 6-DOF parallel manipulators using parallelepiped mechanisms. IEEE Transactions on Robotics 21(5), 821-833 (2005) 